[Article]

\title{
$\mathrm{Ce}_{0.8} \mathrm{Nd}_{0.2} \mathrm{O}_{1.9}-\mathrm{La}_{0.95} \mathrm{Sr}_{0.05} \mathrm{Ga}_{0.9} \mathrm{Mg}_{0.1} \mathrm{O}_{3-\delta}$ 固体复合电解质的结构和电性能
}

\author{
汪秀萍 周德风* 杨国程孙世成 李朝辉 \\ (长春工业大学化学与生命科学学院, 长春 130012)
}

\begin{abstract}
摘要：采用溶胶-凝胶法分别制备 $\mathrm{La}_{0.95} \mathrm{Sr}_{0.05} \mathrm{Ga}_{0.9} \mathrm{Mg}_{0.1} \mathrm{O}_{3-\delta}$ ( $\mathrm{LSGM}$ )和 $\mathrm{Ce}_{0.8} \mathrm{Nd}_{0.2} \mathrm{O}_{1.9}(\mathrm{NDC})$ 电解质, 并在 $\mathrm{NDC}$ 溶 胶中加入 0-15\% ( $w$, 质量分数)的 LSGM 预烧粉体制得 NDC-LSGM复合电解质, 研究不同质量比复合电解质 的结构和电性能. 采用 X射线衍射(XRD)、场发射扫描电子显微镜(FE-SEM)和X能量色散谱仪(EDS)对样品进 行结构表征, 交流(AC)阻抗谱测试样品导电性能. 结果表明: NDC-LSGM复合体系主要由立方萤石结构相、䥻 铁矿结构相和杂质相组成; LSGM 的添加可促进晶粒的生长, 产生大量相界面, 清除或降低 $\mathrm{SiO}_{2}$ 有害影响, 明显 提高晶界导电性; LSGM 质量分数为 $10 \%$ 的样品 NL10 具有最高晶界电导率和总电导率, $400{ }^{\circ} \mathrm{C}$ 时 NL10 的晶 界电导率 $\sigma_{\mathrm{gb}}$ 和总电导率 $\sigma_{\mathrm{t}}$ 分别为 $12.15 \times 10^{-4}$ 和 $3.49 \times 10^{-4} \mathrm{~S} \cdot \mathrm{cm}^{-1}$, 与 $N D C$ 的 $\sigma_{\mathrm{gb}}\left(1.41 \times 10^{-4} \mathrm{~S} \cdot \mathrm{cm}^{-1}\right)$ 和 $\sigma_{\mathrm{t}}(1.20 \times$ $10^{-4} \mathrm{~S} \cdot \mathrm{cm}^{-1}$ 相比分别提高了 7.62 和 1.91 倍, 总电导率的提高主要归因于晶界电导率的影响.
\end{abstract}

关键词：复合电解质；掺钕氧化铈；交流阻抗谱；晶界电导率；镓酸镧 中图分类号: $\mathrm{O} 649$

\section{Structure and Electrical Properties of $\mathrm{Ce}_{0.8} \mathrm{Nd}_{0.2} \mathrm{O}_{1.9}-\mathrm{La}_{0.95} \mathrm{Sr}_{0.05} \mathrm{Ga}_{0.9} \mathrm{Mg}_{0.1} \mathrm{O}_{3-\delta}$ Solid Composite Electrolytes}

\author{
WANG Xiu-Ping ZHOU De-Feng* YANG Guo-Cheng SUN Shi-Cheng LI Zhao-Hui \\ (School of Chemistry and Life Science, Changchun University of Technology, Changchun 130012, P. R. China)
}

\begin{abstract}
Ce}_{0.8} \mathrm{Nd}_{0.2} \mathrm{O}_{1.9}(\mathrm{NDC})$ and $\mathrm{La}_{0.95} \mathrm{Sr}_{0.05} \mathrm{Ga}_{0.9} \mathrm{Mg}_{0.1} \mathrm{O}_{3-\delta}(\mathrm{LSGM})$ electrolytes were each prepared using a sol-gel method. NDC-LSGM composite electrolytes were then prepared by adding $0-15 \%$ ( $w$, mass fraction) precalcined LSGM powders to NDC sols. The microstructure and phase composition of the pellets were characterized using X-ray diffraction (XRD), field-emission scanning electron microscopy (FE-SEM), and energydispersive X-ray spectroscopy (EDS). The electrical conductivities of the pellets were measured using alternative current (AC) impedance spectroscopy. The results showed that all the composites were composed of the cubic fluorite structure, perovskite structure, and secondary phases. The LSGM additive significantly promoted grain growth. The grain boundary conduction increased greatly as a result of the presence of phase interfaces and mitigation of the harmful effects of $\mathrm{SiO}_{2}$ impurities. NL10 was found to have the highest conductivities $\left(\sigma_{g \mathrm{~g}}=12.15 \times\right.$ $10^{-4} \mathrm{~S} \cdot \mathrm{cm}^{-1}, \sigma_{\mathrm{t}}=3.49 \times 10^{-4} \mathrm{~S} \cdot \mathrm{cm}^{-1}$ at $400{ }^{\circ} \mathrm{C}$ ); these values are 7.62 and 1.91 times higher than those of NDC $\left(\sigma_{\mathrm{gb}}=1.41 \times 10^{-4} \mathrm{~S} \cdot \mathrm{cm}^{-1}, \sigma_{\mathrm{t}}=1.2 \times 10^{-4} \mathrm{~S} \cdot \mathrm{cm}^{-1}\right)$. The enhancement of the total conductivity of NL10 is mainly attributed to the large increase in grain boundary conductivity.
\end{abstract}

Key Words: Composite electrolyte; Neodymium-doped ceria; AC impedance spectroscopy; Grain boundary conductivity; Lanthanum gallate

Received: August 11, 2013; Revised: November 11, 2013; Published on Web: November 14, 2013.

*Corresponding author. Email: defengzhou65@126.com; Tel: +86-431-85717276.

The project was supported by the National Natural Science Foundation of China (20871023) and Science Research Foundation of Jilin Province, China (20101549, 20130102001JC).

国家自然科学基金(20871023)和吉林省科技发展计划(20101549, 20130102001JC)资助项目

(C) Editorial office of Acta Physico-Chimica Sinica 


\section{1 引言}

$\mathrm{CeO}_{2}$ 基立方萤石材料由于在高温具有较高的 离子导电性, 与阴极材料间有较好的兼容性, 被认 为是最有希望的中温固体氧化物燃料电池(IT$\mathrm{SOFC}$ ) 电解质. ${ }^{1-3}$ 在 $\mathrm{CeO}_{2}$ 中掺杂二价碱土金属氧化 物或三价稀土金属氧化物, 由于电荷补偿而使氧空 位浓度增加, 从而提高其离子导电性. ${ }^{4,5}$ 然而, $\mathrm{CeO}_{2}$ 基材料在还原性气氛中 $\left(p_{\mathrm{O}_{2}} \leqslant 10^{-10} \mathrm{~Pa}\right), \mathrm{Ce}^{4+}$ 容易被 部分还原为 $\mathrm{Ce}^{3+}$ 产生电子导电, 因抵消部分离子电 导而降低电池的输出功率密度. ${ }^{6,7}$

设计复合电解质或双层电解质可以抑制或克 服电子电导的产生. ${ }^{8-13}$ 如功能复合电解质 $\mathrm{CeO}_{2}$ 基$\mathrm{Li}_{2} \mathrm{CO}_{3} / \mathrm{Na}_{2} \mathrm{CO}_{3} / \mathrm{K}_{2} \mathrm{CO}_{3}$ 中, 熔融的碳酸盐高度分布在 $\mathrm{CeO}_{2}$ 基晶粒周边, 形成特殊的界面结构, 可为离子 传输提供“超离子高速公路”, 因而具有较高的离子 导电性, ${ }^{8-10}$ 如在掺杂 $\mathrm{CeO}_{2}$ 基材料与阳极材料之间加 入掺杂 $\mathrm{ZrO}_{2}$ 基薄膜, 形成掺杂的 $\mathrm{CeO}_{2} / \mathrm{ZrO}_{2}$ 双层电 解质可以抑制 $\mathrm{Ce}^{4+}$ 的还原; ${ }^{11} \mathrm{Y}_{2} \mathrm{O}_{3}$ 掺杂的 $\mathrm{ZrO}_{2}(\mathrm{YSZ})$ 薄膜充当 $\mathrm{Gd}_{2} \mathrm{O}_{3}$ 掺杂的 $\mathrm{CeO}_{2}(\mathrm{GDC})$ 中电子导电的阻 碍层, 能有效提高 YSZ/GDC 双层电解质电池的开 路电压 $(\mathrm{OCV})$. $^{12,13}$ 然而双层电解质电池在长期的工 作过程中, 因界面发生内部扩散而降低其电导率. ${ }^{14}$ $\mathrm{SrO}$ 和 $\mathrm{MgO}$ 共掺杂的 $\mathrm{LaGaO}_{3}$ (LSGM) 具有较好的 离子导电性, ${ }^{15-17}$ 但 LSGM 电解质与常用的阳极材料 $\left(\mathrm{NiO}_{3} 、 \mathrm{Co}_{2} \mathrm{O}_{3} 、 \mathrm{Fe}_{2} \mathrm{O}_{3}\right)$ 之间兼容性较差, 易发生扩散生 成低导电相 $\mathrm{LaNiO}_{3} 、 \mathrm{LaFeO}_{3} 、 \mathrm{LaCoO}_{3}{ }^{18}$ 由于 $\mathrm{LSGM}$ 在宽氧分压范围内电子电导率可忽略不计, 因此可 充当电子阻塞相能有效抑制 $\mathrm{CeO}_{2}$ 中的电子导电. ${ }^{19}$ $\mathrm{Liu}$ 等 ${ }^{20}$ 制备 $\mathrm{Sm}_{2} \mathrm{O}_{3}$ 掺杂的 $\mathrm{CeO}_{2}$ (SDC)-LSGM 复合 电解质, SDC-LSGM 质量比为 9:1 时导电性明显高 于单相 $\mathrm{SDC}$ 电解质, $750{ }^{\circ} \mathrm{C}$ 时其功率密度是 $\mathrm{SDC}$ 的 两倍. Kim 等 ${ }^{21}$ 研究 GDC-LSGM 复合电解质, GDC 与 LSGM 质量比为 50:50 和 80:20 时, 电子电导率随 着氧分压的降低而增加的幅度明显较纯 GDC 电解 质小, GDC 与 LSGM 质量比为 90:10 时拥有最大离 子电导率. 最近, Liu 等 ${ }^{22}$ 将不同质量比的 SDCLSGM复合电解质制成单电池, SDC-LSGM 质量比 为 95:5 时其电流密度和开路电压均明显高于 SDC 单相电解质和 SDC-LSGM 质量比为 90:10 的复合电 解质. 由此可见, 在 $\mathrm{CeO}_{2}$ 基电解质的基础上掺入 LSGM 第二相进行复合改性研究, 可综合发挥各组 分电解质材料的优点, 克服单一组分电解质的缺 点, 而获得高性能的复合电解质材料. 而有关 $\mathrm{Nd}_{2} \mathrm{O}_{3}$
掺杂的 $\mathrm{CeO}_{2}(\mathrm{NDC})$ 与 $\mathrm{LSGM}$ 复合电解质研究的报 道还很少见. 因此, 本文采用溶胶-凝胶法合成 $\mathrm{La}_{0.95} \mathrm{Sr}_{0.05} \mathrm{Ga}_{0.9} \mathrm{Mg}_{0.1} \mathrm{O}_{3-\delta}$ (LSGM) 粉体, 将 LSGM 粉体 加入到 $\mathrm{Ce}_{0.8} \mathrm{Nd}_{0.2} \mathrm{O}_{1.9}$ (NDC) 溶胶中, 制备不同质量比 的 NDC-LSGM 复合电解质; 讨论不同质量比的 LSGM 对复合电解质结构和电性能的影响.

\section{2 实验部分}

\section{1 材料的制备}

以分析纯的 $\mathrm{La}\left(\mathrm{NO}_{3}\right)_{3} \cdot 6 \mathrm{H}_{2} \mathrm{O}(99.5 \%(w$, 下同)), $\mathrm{Sr}\left(\mathrm{NO}_{3}\right)_{2}(99.5 \%), \mathrm{Mg}\left(\mathrm{NO}_{3}\right)_{2} \cdot 6 \mathrm{H}_{2} \mathrm{O}(99.0 \%)$ 和高纯 $\mathrm{Ga}(99.999 \%$, Aladdin) 为起始原料, 按化学计量比 $\mathrm{La}_{0.95} \mathrm{Sr}_{0.05} \mathrm{Ga}_{0.9} \mathrm{Mg}_{0.1} \mathrm{O}_{3-\delta}$ 精确称量以上原料, $\mathrm{Ga}$ 用硝酸 溶解后加入反应体系中, 然后加入金属离子物质的 量 1.5 倍的柠檬酸和少量的聚乙二醇(PEG)20000, 加热搅拌形成透明溶胶后转移到陶瓷蒸发血.中, $70-80{ }^{\circ} \mathrm{C}$ 水浴蒸发约 $12 \mathrm{~h}$ 形成干凝胶, 在 $1000{ }^{\circ} \mathrm{C}$ 预烧 $10 \mathrm{~h}$ 除去残留有机物. 然后将上述粉体在玛瑙 研针中研磨成更细粉末.

以分析纯的 $\mathrm{Ce}\left(\mathrm{NO}_{3}\right)_{3} \cdot 6 \mathrm{H}_{2} \mathrm{O}(99.5 \%)$ 和高纯 $\mathrm{Nd}_{2} \mathrm{O}_{3}(99.99 \%$, 上海国药集团)为原料, 按化学计量 比 $\mathrm{Ce}_{0.8} \mathrm{Nd}_{0.2} \mathrm{O}_{1.9}$ 精确称量以上原料. $\mathrm{Nd}_{2} \mathrm{O}_{3}$ 用硝酸溶 解后加入柠檬酸和聚乙二醇, 搅拌 2-3 h 待形成透 明溶胶后, 按 NDC:LSGM 的质量比 95:05、90:10 和 $85: 15$ 的比例加入预烧的 LSGM 粉末(样品分别标记 为 NL05、NL10 和 NL15), 磁力搅拌器上搅拌均匀. 制得的干凝胶在 $550{ }^{\circ} \mathrm{C}$ 预烧 $10 \mathrm{~h}$, 然后将上述复合 粉体在玛瑙研钵中研磨并于 $10 \mathrm{MPa}$ 下压成直径约 $13 \mathrm{~mm}$ 、厚度约 $1.3 \mathrm{~mm}$ 的圆片, 于程序控制高温炉 中 $1400{ }^{\circ} \mathrm{C}$ 烧结 $10 \mathrm{~h}$ 后自然冷却至室温. 上述样片 经抛光清洗后在两面涂上银胶, 经 $550{ }^{\circ} \mathrm{C}$ 加热 $1 \mathrm{~h}$ 用于电化学性能测试.

\section{2 材料的结构表征与电性能测试}

采用日本理学 D/MAX2000/PC 型 X 射线衍射 仪 $\left(\mathrm{Cu} K_{\mathrm{a} 1}, \lambda=0.15405 \mathrm{~nm}\right)$ 测定样品的 XRD 图谱, 工 作电压 $40 \mathrm{kV}$, 工作电流 $40 \mathrm{~mA}$, 扫描速率为 $1\left(^{\circ}\right)$. $\mathrm{min}^{-1}$; 使用德国赛斯公司的 SUPPA40场发射扫描电 子显微镜(FE-SEM)和美国 EDAX-Falcon型 X 能量 色散谱仪 (EDS) 观测样品微观形貌及分析相组 成; 利用美国 PARSTAT2273 频响分析仪测定样品 在 300-800 ${ }^{\circ} \mathrm{C}$ 升温程序下的阻抗谱(温度间隔为 $50{ }^{\circ} \mathrm{C}$ ), 频率范围 $0.1 \mathrm{~Hz}-1.0 \mathrm{MHz}$, 交流微扰电压为 $10 \mathrm{mV}$, 温度由 UGB2805 型自控温度仪控制; 用 
ZSimpWin 软件分析待测样品的电阻 $(R)$, 模拟阻抗 谱图, 根据样品厚度 $(D)$ 和表面积 $(S)$ 计算电导率, 计 算公式为 $\sigma=D /(R \cdot S)$.

\section{3 结果与讨论}

\subsection{XRD 分析}

图 1 是 LSGM 预烧粉体以及 NDC、NL05、 NL10、NL15 和 LSGM 烧结样片的 XRD 谱图. 由图 可知, LSGM 预烧粉体与 LSGM 烧结样片一样均为 钻钛矿结构, 用 Jade 软件计算 LSGM 粉体在(112)晶 面的粒径约为 $30 \mathrm{~nm}$. 复合样品中主要出现 8 条立方 䒯石结构的衍射峰, 说明复合体系主要由 NDC 组 成. 图 1 中插图为复合电解质在衍射角 $2 \theta$ 为 $31^{\circ}-41^{\circ}$ 范围内的放大 XRD 谱图. 由图中可知, NL15样品在 衍射角为 $2 \theta=32^{\circ}$ 和 $40^{\circ}$ 附近均出现了钙钛矿结构的 特征峰, 而 NL10 和 NL05 样品也在 $2 \theta=40^{\circ}$ 附近出现 钙钛矿结构的特征峰, 由此说明复合体系均存在 LSGM 钙钛矿结构相. 另外, 插图中还出现了未知杂 质相的衍射峰, 由杂质相衍射峰强度变化可知, 杂 质相含量随着 LSGM 含量的增加而增加. 杂质相的 存在将会影响复合体系的电导率. 图 1 中复合电解 质的衍射峰与单纯的 NDC 相比明显增强, 说明复合 体系中 NDC 的结晶度明显增加. 由图 2 给出的 NDC 晶胞参数与 LSGM 含量的关系图可知, 复合电解质 的晶胞参数随 LSGM 含量的增加而略微增加, 可能 是由于在 LSGM 钙钛矿相与 NDC 萤石相中离子存

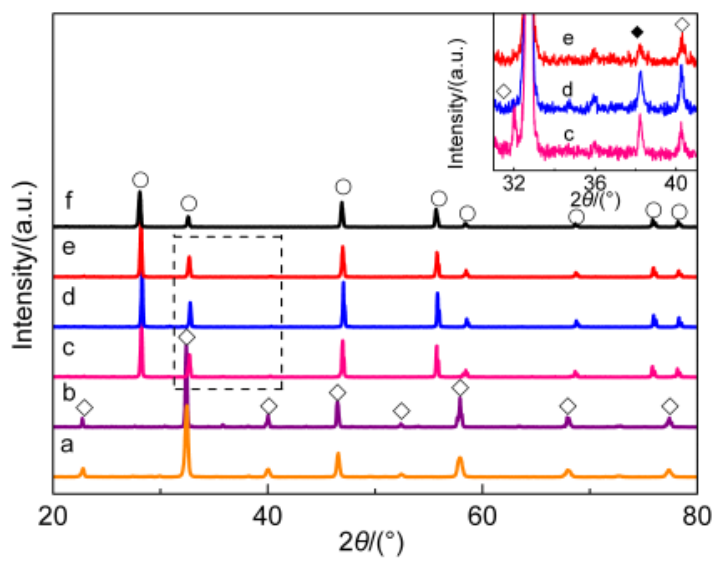

图 1 样品的XRD 谱图

Fig.1 XRD patterns of the samples (a) LSGM powder, (b) LSGM, (c) NL15, (d) NL10, (e) NL05, (f) NDC; $\diamond$ LSGM, ONDC, unknown phase; LSGM: $\mathrm{La}_{0.95} \mathrm{Sr}_{0.05} \mathrm{Ga}_{0.9} \mathrm{Mg}_{0.1} \mathrm{O}_{3-\delta}, \mathrm{NDC}: \mathrm{Ce}_{0.8} \mathrm{Nd}_{0.2} \mathrm{O}_{1.9}$; the composite electrolytes with NDC to LSGM mass ratios of 95:5, 90:10, and 85:15 are refered to as NL05, NL10, and NL15, respectively.

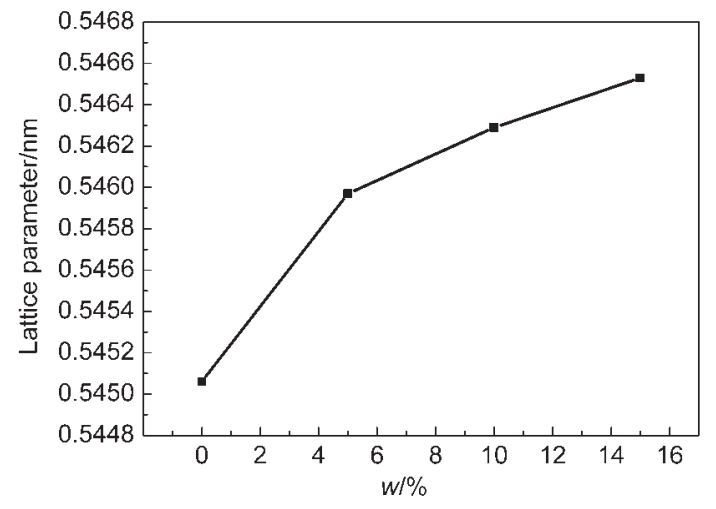

图 $2 \mathrm{NDC}$ 晶胞参数与 LSGM 质量分数 $(w)$ 的关系

Fig.2 Lattice parameter of NDC as a function of LSGM mass fraction ( $w)$

在不同的化学势而发生了离子扩散. ${ }^{23}$ 由于六配位 $\mathrm{La}^{3+}$ 离子的半径 $(0.116 \mathrm{~nm})$ 略大于六配位 $\mathrm{Ce}^{4+}$ 离子的 半径 $(0.097 \mathrm{~nm}), \mathrm{La}^{3+}$ 离子进入 $\mathrm{NDC}$ 晶格会引起晶胞 体积增加. 且随着 LSGM 含量的增加, 可能会有更 多的 $\mathrm{La}^{3+}$ 离子固溶到 NDC 体系中, 所以晶胞参数也 随之增加..$^{24}$

\section{2 样品的微观形貌分析}

图 3 是 $1400^{\circ} \mathrm{C}$ 烧结 $10 \mathrm{~h}$ 的 FE-SEM 图和 EDS 图. 在复合体系中均出现微小黑色颗粒, 如图中三 角形标记区域, 由 EDS 图(如图 3)可知, 黑色颗粒为 $\mathrm{Ga}$ 丰富相, 这可能是由于 $\mathrm{La}^{3+}$ 离子发生扩散, 破坏 了LSGM钙钛矿结构而生成含 $\mathrm{Ga}$ 丰富相. 由于黑色 颗粒太小且低于 EDS 检测限度, 因而无法确定其组 成. 且由图 3 可知, $\mathrm{Ga}$ 丰富相随着 LSGM 含量的增 加而增加, 与图 1XRD 分析结果一致, 由此可推测 XRD 图中出现的未知杂质相可能是 $\mathrm{Ga}$ 丰富相. 除 了黑色颗粒外, 复合体系中的白色微小颗粒如圆形 标记区域, 可能是 LSGM 相, 且随着设计的 LSGM 含量的增加而增加. 另外, 复合体系晶粒均大于 NDC 纯体系, 且随着 LSGM 含量的增加晶粒尺寸增 大(图 3(c)的 NL10), 但是当 LSGM 质量分数达 $15 \%$ 时, 晶粒尺寸略微减小, 并有较多气孔存在(如图 3 (d) 的 NL15). 晶粒生长主要与晶格畸变有关. ${ }^{25,26}$ 由 于 $\mathrm{La}^{3+}$ 离子的半径 $(0.116 \mathrm{~nm})$ 大于 $\mathrm{Ce}^{4+}$ 离子的半径 $(0.097 \mathrm{~nm})$, 烧结过程中容易引起晶格畸变, 有利于 缺陷的迁移, 从而促进晶界移动及晶粒生长. 而 NL10 中较多的 LSGM 相、Ga丰富相和气孔可抑制 晶界的迁移, 因此晶粒尺寸相对较小. 由此可知, 少 量 LSGM 的添加有利于晶粒的生长, 即促进烧结; 而 LSGM 含量较多时, 晶粒生长反而受到抑制, 不 利于致密性的提高. 


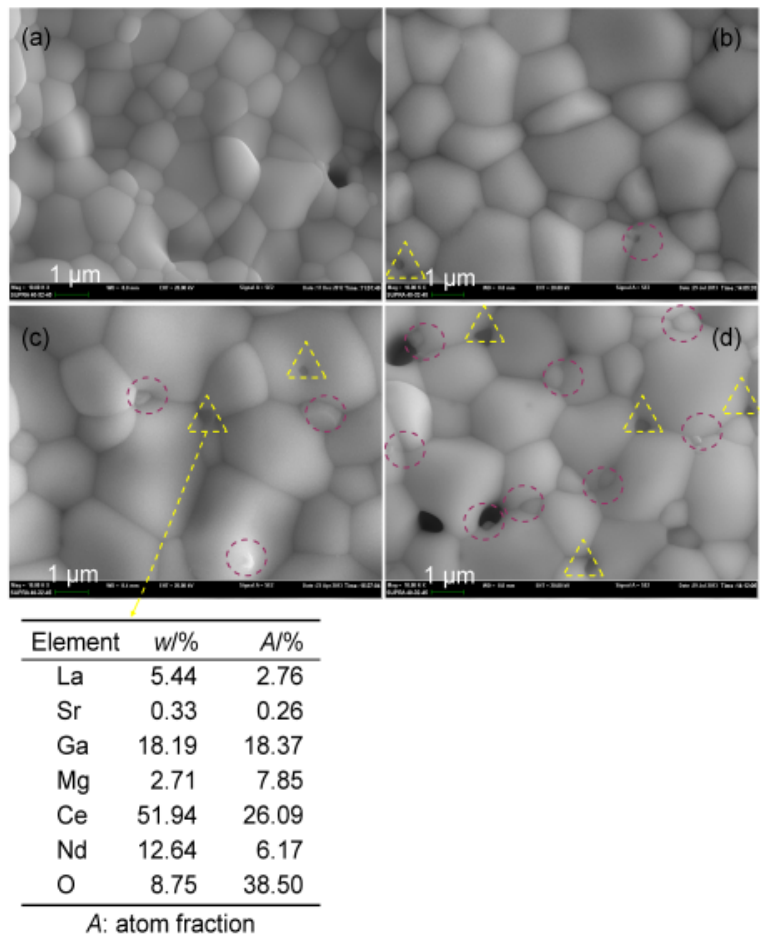

图 3 样品于 $1400{ }^{\circ} \mathrm{C}$ 烧结 $10 \mathrm{~h}$ 后场发射扫描 电镜图及对应区域能谱分析

Fig.3 FE-SEM images and the corresponding EDS spectrum analysis of marked areas of samples sintered at $1400{ }^{\circ} \mathrm{C}$ for $10 \mathrm{~h}$ (a) NDC, (b) NL05, (c) NL10, (d) NL15

\section{3 交流阻抗谱图分析}

标准的交流阻抗谱图通常由三段半圆弧组成, 从高频到低频分别对应于晶粒、晶界和电极界面响 应三个连续的过程. 实际上由于仪器有限的频率范 围以及晶粒、晶界电阻随温度变化不同, 导致测量 中出现不同的谱图. 图 4 为经 $1400{ }^{\circ} \mathrm{C}$ 烧结的所有样 品于 $400{ }^{\circ} \mathrm{C}$ 空气中测试模拟的交流阻抗谱图及等 效电路图. 所有阻抗谱图都由一个半圆和一小段射 线弧组成, 等效于 2 个并联 $R C$ 电路串联构成. 图中 半圆的高频端与实轴的交点代表晶粒电阻 $\left(R_{\mathrm{gi}}\right)$, 半 圆弧在实轴的截距为晶界电阻 $\left(R_{\mathrm{gb}}\right)$, 半圆低频端与 实轴的交点坐标为总电阻 $\left(R_{\mathrm{t}}\right)\left(R_{\mathrm{t}}=R_{\mathrm{gi}}+R_{\mathrm{gb}}\right)$. 结合图 4
和表 1 中 $1400{ }^{\circ} \mathrm{C}$ 烧结样品在测试温度 $400{ }^{\circ} \mathrm{C}$ 时的 电阻、电导率和活化能数据可知, NL05、NL10 和 NL15 复合电解质的晶粒电阻分别为 566.3、305.6 和 $794.0 \Omega$, 均比单相 NDC 的晶粒电阻(184.9 $\Omega$ )大, 原 因是少量离子半径较大的 $\mathrm{La}^{3+}$ 离子进入 $\mathrm{NDC}$ 体系 中引起晶格扭曲, ${ }^{27}$ 从而增加了晶粒电阻. 复合电解 质的晶界电阻分别为 $330.7 、 123.2$ 和 $502.0 \Omega$, 均明 显小于 NDC 晶界电阻 $(1084.1 \Omega)$. 同时从表 1 中可 知, 虽然 NL10 的晶粒电阻 (305.6 $\Omega$ ) 比 NDC 的晶粒 电阻(184.9 $\Omega$ )大, 但是由于其晶界电阻(123.2 $\Omega$ )远 远小于 NDC 的晶界电阻 $(1084.1 \Omega)$, 总的变化结果 是 NDC 与 LSGM 的质量比为 90:10 时的 NL10 具有 最小的总电阻. 因此样品 NL10 可望具有最好的导 电性.

\section{4 电性能分析}

图 5 给出晶粒电导率、晶界电导率和总电导率 与温度的阿仑尼乌斯关系. 从图 5(a) 可知, 在 300$600{ }^{\circ} \mathrm{C}$ 纯 $\mathrm{NDC}$ 的晶粒电导率明显高于复合体系晶 粒电导率. $400^{\circ} \mathrm{C}$ 时 $\mathrm{NDC}$ 的晶粒电导率 $\left(8.25 \times 10^{-4} \mathrm{~S}\right.$.

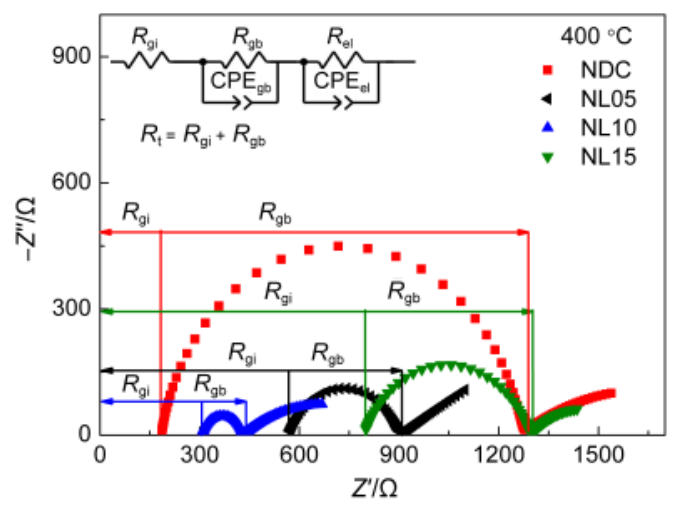

图 4 在 $400{ }^{\circ} \mathrm{C}$ 空气中 NDC 和 NDC-LSGM 样品的 交流阻抗谱图和等效电路

Fig.4 Equivalent circuit and complex impedance spectra of NDC and NDC-LSGM samples measured at $400{ }^{\circ} \mathrm{C}$ in air

$R_{\mathrm{g}}$ : grain resistance; $R_{\mathrm{gb}}$ : grain boundary resistance; $R_{\mathrm{el}}$ : electrode resistance; $\mathrm{CPE}_{\mathrm{gb}}$ : constant phase element of the grain boundary; $\mathrm{CPE}_{\mathrm{el}}$ : constant phase element of the electrode

表 1 NDC 和 NDC-LSGM 烧结样品在测试温度 $400^{\circ} \mathrm{C}$ 时电阻、电导率和活化能

Table 1 Resistances, electrical conductivities, and activation energies of NDC and NDC-LSGM sintered samples measured at $400{ }^{\circ} \mathrm{C}$

\begin{tabular}{|c|c|c|c|c|c|c|c|c|}
\hline Sample & $R_{\mathrm{g} i} / \Omega$ & $R_{\mathrm{gb}} / \Omega$ & $R_{\mathrm{t}} / \Omega$ & $10^{4} \sigma_{\mathrm{g}} /\left(\mathrm{S} \cdot \mathrm{cm}^{-1}\right)$ & $10^{4} \sigma_{\mathrm{gb}} /\left(\mathrm{S} \cdot \mathrm{cm}^{-1}\right)$ & $10^{4} \sigma_{t} /\left(\mathrm{S} \cdot \mathrm{cm}^{-1}\right)$ & $E_{\mathrm{gb}} / \mathrm{eV}$ & $E_{\mathrm{t}} / \mathrm{eV}$ \\
\hline $\mathrm{NDC}$ & 184.9 & 1084.1 & 1269.0 & 8.25 & 1.41 & 1.20 & 1.086 & 1.016 \\
\hline NL05 & 566.3 & 330.7 & 897.0 & 2.45 & 4.19 & 1.55 & 1.057 & 0.912 \\
\hline NL10 & 305.6 & 123.2 & 428.8 & 4.90 & 12.15 & 3.49 & 1.036 & 0.898 \\
\hline NL15 & 794.0 & 502.0 & 1296.0 & 1.80 & 2.85 & 1.10 & 1.131 & 0.926 \\
\hline
\end{tabular}

$\sigma_{\mathrm{g}}, \sigma_{\mathrm{g} b}, \sigma_{\mathrm{t}}$ indicate grain, grain boundary, and total conductivities, respectively. ${ }^{*} E_{\mathrm{gb}}, E_{\mathrm{t}}$ indicate grain boundary and total activation energies, respectively. 
$\left.\mathrm{cm}^{-1}\right)$ 是 NL10 晶粒电导率 $\left(4.90 \times 10^{-4} \mathrm{~S} \cdot \mathrm{cm}^{-1}\right)$ 的 1.68 倍, 说明添加少量 LSGM 于 NDC 体系对材料的晶粒 电导产生不利的影响. 这可能与 $\mathrm{La}^{3+}$ 离子进入 $\mathrm{NDC}$ 体系引起晶胞参数的改变有关. $\mathrm{La}^{3+}$ 离子进入 $\mathrm{NDC}$ 体系中可能会产生两种影响. 其一是少量离子半径 较大的 $\mathrm{La}^{3+}$ 离子 $(0.116 \mathrm{~nm})$ 进入晶格, 引起晶格扭曲 而阻碍氧离子的迁移, ${ }^{27}$ 因而晶粒电导率降低; 其二 是 $\mathrm{La}^{3+}$ 与 $\mathrm{Nd}^{3+}$ 均为 +3 价, 少量 $\mathrm{La}^{3+}$ 离子进入晶格取 代 $\mathrm{Ce}^{4+}$, 产生氧空位 $\left(V_{\mathrm{O}}^{\circ}\right)$ 而使导电性提高, ${ }^{28}$ 同时 $\mathrm{La}^{3+}$ 离子 $(0.116 \mathrm{~nm})$ 与 $\mathrm{Ce}^{4+}(0.097 \mathrm{~nm})$ 的半径不匹配程度 大于 $\mathrm{Nd}^{3+}$ 离子 $(0.111 \mathrm{~nm})$ 与 $\mathrm{Ce}^{4+}$ 离子 $(0.097 \mathrm{~nm})$ 的 半径不匹配程度, 因此 $\mathrm{La}^{3+}$ 离子取代的 $\mathrm{Ce}^{4+}$ 离子 $\left(\mathrm{La}^{\prime}{ }_{\mathrm{Ce}}\right)$ 与 $V_{\mathrm{o}}^{*}$ 之间的电荷作用小于 $\mathrm{Nd}^{3+}$ 离子取代的 $\mathrm{Ce}^{4+}$ 离子 $\left(\mathrm{Nd}^{\prime}{ }_{\mathrm{Ce}}\right)$ 与 $V_{\mathrm{O}}^{* *}$ 之间的电荷作用, 所以 $\mathrm{La}^{\prime}{ }_{\mathrm{Ce}}$ 与 $V_{\mathrm{O}}^{* *}$ 不易结合成缺陷缔合体 $\left[\mathrm{La}^{\prime}{ }_{\mathrm{Ce}} \cdot V_{\mathrm{O}}^{*}\right]$, 则自由 $V_{\mathrm{o}}^{*}$ 增 多, 导电性提高. 我们研究的复合体系 NDC-LSGM 的晶粒导电性的降低可能是第一种因素起主要作 用. 从图 5(a) 可知, NL10 的晶粒电导率 $\left(4.90 \times 10^{-4} \mathrm{~S}\right.$. $\left.\mathrm{cm}^{-1}\right)$ 要明显高于 $\mathrm{NL} 05\left(2.45 \times 10^{-4} \mathrm{~S} \cdot \mathrm{cm}^{-1}\right)$ 和 NL15 $\left(1.80 \times 10^{-4} \mathrm{~S} \cdot \mathrm{cm}^{-1}\right)$ 的晶粒电导率, 可能是与其较大 的晶粒尺寸有关. 晶粒长大, 相应的晶界区域减 小, ${ }^{29}$ 从而晶粒电导率增加. ${ }^{30}$

从图 5(b) 可知, 复合体系的晶界导电性高于 $\mathrm{NDC}$ 体系. 如测试温度为 $400{ }^{\circ} \mathrm{C}$ 时, NL05、NL10 和 $\mathrm{NL} 15$ 的晶界电导率分别为 $4.19 \times 10^{-4} 、 12.15 \times 10^{-4}$ 和 $2.85 \times 10^{-4} \mathrm{~S} \cdot \mathrm{cm}^{-1}$, 与 $\mathrm{NDC}$ 的晶界电导率 $\left(1.41 \times 10^{-4} \mathrm{~S} \cdot\right.$ $\mathrm{cm}^{-1}$ ) 相比分别提高了 1.97、7.62 和 1.04 倍, 其中 NL10 晶界导电性提高最为明显, 且具有最小晶界 活化能(1.036 eV). NDC-LSGM复合体系的晶界导
电性大大提高可能与界面导电机理有关. ${ }^{31} \mathrm{NDC}$ 为 立方萤石结构, LSGM 为钙钛矿结构, 两相之间的晶 体结构和晶胞参数之间存在较大差异, 在两相界面 处可能会产生较大的晶格畸变, 进而产生大量位错 等晶体缺陷, 而离子的迁移率在富位错区域或者高 缺陷浓度区域要远远高于理想晶体, 32 因此 NDC 和 LSGM 两相界面的存在有利于晶界导电性的提高. 另外, 对于 $\mathrm{CeO}_{2}$ 或 $\mathrm{ZrO}_{2}$ 固体电解质而言, $\mathrm{SiO}_{2}$ 杂质 相在制备和烧结过程中很容易引入体系而增大晶 界电阻, 导致晶界电导率下降. 电感偶合等离子体 (ICP) 测量预烧 NDC 粉体在玛瑙研钵中研磨 $1 \mathrm{~h}$ 后, 其中主要杂质 $\mathrm{SiO}_{2}$ 的含量约为 $0.003 \%(\omega)(<0.05 \%$ $(\omega))$, 年属于高纯体系. $\mathrm{SiO}_{2}$ 的存在是阻碍晶界氧离子 传导的一个重要的因素. 3 . 氧离子只能通过 “干净的 晶粒一晶粒” 接触穿过晶界来传输, ${ }^{35} \mathrm{SiO}_{2}$ 杂质相的存 在减小了这种“干净的晶粒-晶粒”接触的区域, 阻碍 氧离子的传输. 本文中添加 LSGM 后晶界电阻大大 减少, 可能是由于 $\mathrm{LSGM}$ 能够促进 $\mathrm{SiO}_{2}$ 在晶界相的 析出, 增强了晶粒与晶粒之间的接触, ${ }^{36}$ 相应的阻碍 氧空位传输的晶界区域会减少, 产生了比较好的晶 界电导率; 也可能是由于其中含有碱土金属离子 $\mathrm{Sr}^{2+}$ 和 $\mathrm{Mg}^{2+}$, 与酸性 $\mathrm{SiO}_{2}$ 有较好的化学亲和力生成导 电性好的 $\mathrm{Mg}_{2} \mathrm{SiO}_{4}$ 或含 $\mathrm{Sr}$ 相而达到清除 $\mathrm{SiO}_{2}$ 的作 用. ${ }^{37,38}$ 在整个测试温度范围内, NL15 晶界导电性提 高幅度最小, 总电导率最低, 可能与其较多的杂质和 气孔有关(图3(d)). 杂质和气孔增加了氧离子在晶界 迁移的能垒, 因此 NL15 具有最大晶界活化能(1.131 $\mathrm{eV}$ ), 晶界电导率降低. 综上所述, NDC 与 LSGM 的 质量比为 90:10 时能大大改善其晶界导电性.
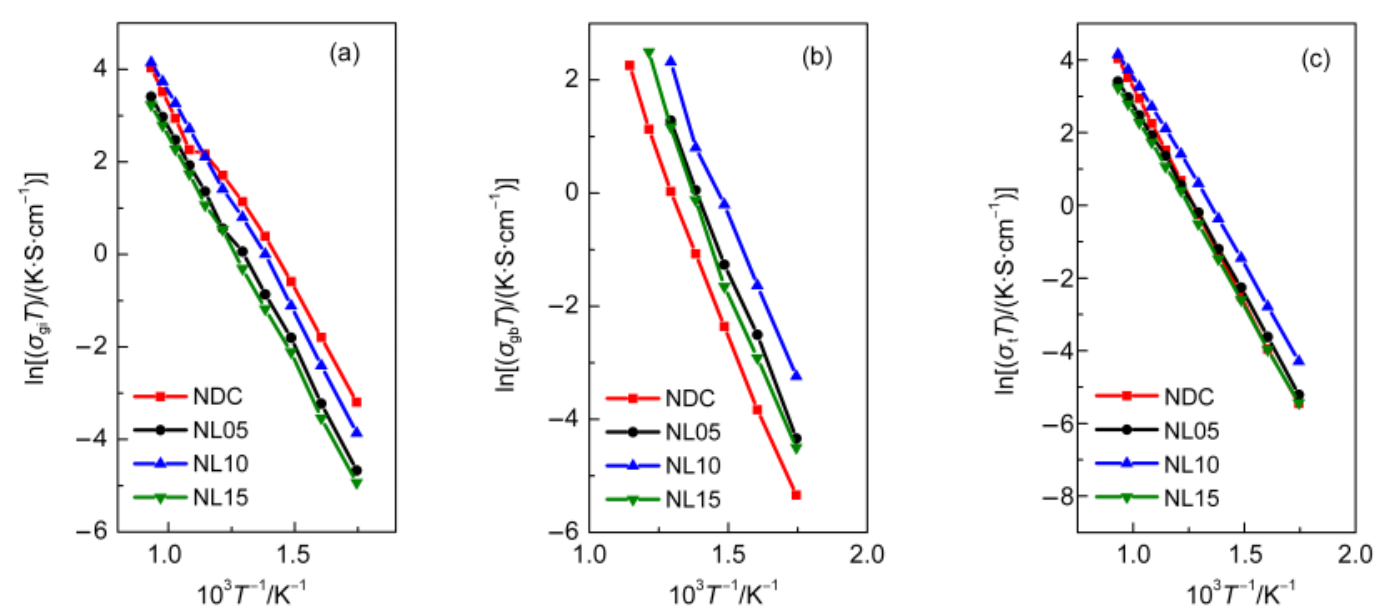

图 5 经 $1400{ }^{\circ} \mathrm{C}$ 烧结后 NDC 和 NDC-LSGM 样品的阿仑里乌斯曲线

Fig.5 Arrhenius plots of NDC and NDC-LSGM samples sintered at $1400{ }^{\circ} \mathrm{C}$ 
分析图 5(c)和表 1 可知, 随着 LSGM 含量的增 加, 体系总电导率逐渐增加, 总活化能 $\left(E_{\mathrm{t}}\right)$ 减小. LSGM 质量分数为 $10 \%$ 的 NL10 具有最大的总电导 率和最小总活化能, 随着 LSGM 含量增加到 $15 \%$ (w) 时, 总电导率明显降低, 总活化能增加. 如测试温 度 $400{ }^{\circ} \mathrm{C}$ 时 $\mathrm{NL} 10$ 的 $\sigma_{\mathrm{t}}$ 为 $3.49 \times 10^{-4} \mathrm{~S} \cdot \mathrm{cm}^{-1}$, 相比于 $\mathrm{NDC}$ 的 $\sigma_{\mathrm{t}}\left(1.20 \times 10^{-4} \mathrm{~S} \cdot \mathrm{cm}^{-1}\right)$ 提高了 1.91 倍, NL10 的 $E_{\mathrm{t}}$ 为 $0.898 \mathrm{eV}$, 相比于 $\mathrm{NDC}$ 的 $E_{\mathrm{t}}(1.016 \mathrm{eV})$ 明显减 小, 而与 90:10 (w) 的复合体系 $\mathrm{Ce}_{0.8} \mathrm{Sm}_{0.2} \mathrm{O}_{1.9}-\mathrm{La}_{0.9} \mathrm{Sr}_{0.1}$ $\mathrm{Ga}_{0.8} \mathrm{Mg}_{0.2} \mathrm{O}_{2.85}$ 的 $E_{\mathrm{t}}(0.900 \mathrm{eV})$ 相比差别不大; ${ }^{21} 750{ }^{\circ} \mathrm{C}$ 时 NL10 的 $\sigma_{\mathrm{t}}$ 为 $0.041 \mathrm{~S} \cdot \mathrm{cm}^{-1}$, 明显高于 $\mathrm{Ce}_{0.8} \mathrm{Sm}_{0.2} \mathrm{O}_{1.9}$ $\left(0.020 \mathrm{~S} \cdot \mathrm{cm}^{-1}\right)$ 和 90:10 (质量比) 的复合体系 $\mathrm{Ce}_{0.8} \mathrm{Sm}_{0.2} \mathrm{O}_{1.9}-\mathrm{La}_{0.9} \mathrm{Sr}_{0.1} \mathrm{Ga}_{0.8} \mathrm{Mg}_{0.2} \mathrm{O}_{2.85}\left(0.026 \mathrm{~S} \cdot \mathrm{cm}^{-1}\right)$. ${ }^{21}$ 说 明在 NDC 中加入 LSGM 的最适质量比为 90:10. 多 晶材料中总导电率由晶粒电导率和晶界电导率共 同组成. 结合图 5(a) 和 5(b) 可知, NL10 晶界电导率 的提高幅度 (7.62 倍)明显大于晶粒电导率的减小幅 度 (0.41 倍), 因此晶界电导率大大提高是 NL10 样品 拥有最高电导率的主要原因.

\section{4 结 论}

采用溶胶-凝胶法分别制备了 NDC 和 LSGM 电 解质, 在 NDC 溶胶中掺入 LSGM 预烧粉体制得不同 质量比(NDC 与 LSGM 的质量比分别为 95:05、90:10 和 85:15)的复合电解质材料. NDC-LSGM复合体系 主要由立方萤石结构相和钙钛矿结构相和杂质相 组成, 随着 LSGM 加入量的增加其复合体系中 NDC 的晶胞参数增大; LSGM 的添加均可明显促进晶粒 的生长, 引入大量相界面, 清除或降低 $\mathrm{SiO}_{2}$ 有害影 响, 提高晶界导电性. $400{ }^{\circ} \mathrm{C}$ 时 NL05、NL10 和 NL15 的晶界电导率分别为 $4.19 \times 10^{-4} 、 12.15 \times 10^{-4}$ 和 $2.85 \times$ $10^{-4} \mathrm{~S} \cdot \mathrm{cm}^{-1}$, 与 $\mathrm{NDC}$ 晶界电导率 $\left(1.41 \times 10^{-4} \mathrm{~S} \cdot \mathrm{cm}^{-1}\right)$ 相比分别提高了 1.97 、7.62 和 1.04 倍; NL10 具有最 高总电导率, 其晶界电导率的提高幅度(7.62 倍)明 显大于晶粒电导率的减小幅度 $(0.41$ 倍), 是其总电 导率提高的主要原因.

\section{References}

(1) M-Hernandez, A.; V-Castillo, J.; Mogni, L.; Caneiro, A. Int. J. Hydrog. Energy 2011, 36 (24), 15704.

(2) Hart, N. T.; Brandon, N. P.; Day, M. J.; Lapeña-Rey, N. J. Power Sources 2002, 106 (1-2), 42. doi: 10.1016/S0378-7753 (01)01035-7

(3) Steele, B. C. H. Solid State Ionics 2000, 129 (1-4), 95. doi:
10.1016/S0167-2738(99)00319-7

(4) Yan, D. Y.; Liu, X. M.; Bai, X. Y.; Pei, L.; Zheng, M. Z.; Zhu, C. J.; Su, W. H. J. Power Sources 2010, 195, 6488.

(5) Molenda, J.; Świerczek, K.; Zaj c, W. J. Power Sources 2007, $173(2), 660$. ą

(6) Badwal, S. P. S.; Ciacchi, F. T.; Drennan, J. Solid State Ionics 1999, 121 (1-4), 253. doi: 10.1016/S0167-2738(99)00044-2

(7) Abrantes, J. C. C.; Pérez-Coll, D.; Núñez, P.; Frade, J. R. Electrochim. Acta 2003, 48 (19), 2761. doi: 10.1016/S0013-4686 (03)00395-5

(8) Xia, Y. J.; Bai, Y. J.; Wu, X. J.; Zhou, D. F.; Liu, X. J.; Meng, J. Int. J. Hydrog. Energy 2011, 36, 6840. doi: 10.1016/j. ijhydene.2011.02.118

(9) Zhao, Y. C.; Xu, Z. R.; Xia, C.; Li, Y. D. Int. J. Hydrog. Energy 2013, 38, 1553. doi: 10.1016/j.jhydene.2012.11.004

(10) Fan, L. D.; Wang, C. Y.; Chen, M. M.; Zhu, B. J. Power Sources 2013, 234, 154. doi: 10.1016/j.jpowsour.2013.01.138

(11) Kwon, T. H.; Lee, T.; Yoo, H. I. Solid State Ionics 2011, 195 (1), 25. doi: 10.1016/j.ssi.2011.05.002

(12) Cho, S.; Kim, Y. N.; Kim, J. H.; Manthiram, A.; Wang, H. Y. Electrochim. Acta 2011, 56 (16), 5472. doi: 10.1016/j. electacta.2011.03.039

(13) Jang, W. S.; Hyun, S. H.; Kim, S. G. J. Mater. Sci. 2002, 37 (12), 2535. doi: 10.1023/A:1015451910081

(14) Inoue, T.; Setoguchi, T.; Eguchi, K.; Arai, H. Solid State Ionics 1989, 35 (3-4), 285. doi: 10.1016/0167-2738(89)90310-X

(15) Ishihara, T.; Matsuda, H.; Takita, Y. J. Am. Chem. Soc. 1994, 116, 3801. doi: 10.1021/ja00088a016

(16) Huang, K.; Tichy, R. S.; Goodenough, J. B. J. Am. Ceram. Soc. 1998, 81, 2565. doi: 10.1111/j.1151-2916.1998.tb02662.x

(17) Kharton, V. V.; Marques, F. M. B.; Atkinson, A. Solid State Ionics 2004, 174 (1-4), 135. doi: 10.1016/j.ssi.2004.06.015

(18) Zhang, X. G.; Ohara, S.; Okawa, H.; Maric, R.; Fukui, T. Solid State Ionics 2001, 139 (1-2), 145. doi: 10.1016/S0167-2738(00) 00833-X

(19) Xu, D.; Liu, X. M.; Zhu, C. J.; Wang, D. J.; Yan, D. T.; Wang, D. Y.; Su, W. H. J. Rare Earth 2008, 26, 241. doi: 10.1016/ S1002-0721(08)60073-3

(20) Xu, D.; Liu, X. M.; Wang, D. J.; Yi, G. Y.; Gao, Y.; Zhang, D. S.; Su, W. H. J. Alloy. Compd. 2007, 429, 292. doi: 10.1016/j. jallcom.2006.04.009

(21) Jo, S. H.; Muralidharan, P.; Kim, D. K. J. Alloy. Compd. 2010, 491, 416. doi: 10.1016/j.jallcom.2009.10.207

(22) Hao, G. Y.; Liu, X. M.; Wang, H. P.; Be, H. L.; Pei, L.; Su, W. H. Solid State Ionics 2012, 225, 81. doi: 10.1016/j. ssi.2012.03.005

(23) Medvedev, D.; Maragou, V.; Pikalova, E.; Demin, A.; Tsiakaras, P. J. Power Sources 2013, 221, 217. doi: 10.1016/j. jpowsour.2012.07.120

(24) Xu, D.; Liu, X. M.; Wang, D. J.; Zhu, C. J.; Yan, D. T.; Pei, L.; Su, W. H. Chem. J. Chin. Univ. 2008, 29, 1523. [徐 丹, 刘晓 
梅, 王德军, 朱成军, 严端廷, 裴 力, 苏文辉. 高等学校化学学 报, 2008, 29, 1523.]

(25) Zhang, T. S.; Hing, P.; Huang, H. T.; Kilner, J. J. Eur. Ceram. Soc. 2002, 22, 27. doi: 10.1016/S0955-2219(01)00240-0

(26) Li, J. G.; Ikegami, T.; Mori, T. Acta Materialia 2004, 52 (8), 2221. doi: 10.1016/j.actamat.2004.01.014

(27) Pikalova, E. Yu. Murashkina, A. A.; Maragou, V. I.; Demin, A. K.; Strekalovsky, V. N.; Tsiakaras, P. E. Int. J. Hydrog. Energy 2011, 36 (10), 6175. doi: 10.1016/j.ijhydene.2011.01.132

(28) Kahlauoi, M.; Inoubli, A.; Chefi, S.; Kouki, A.; Madani, A.; Chefi, C. Ceram. Int. 2013, 39 (6), 6175. doi: 10.1016/j. ceramint.2013.01.036

(29) Zhou, D. F.; Zhu, J. X.; Xia, Y. J.; Zhao, G. C.; Meng, J. Chin. J. Inorg. Chem. 2010, 26 (1), 91. [周德风, 朱建新, 夏燕杰, 赵桂 春, 孟 健. 无机化学学报, 2010, $26(1), 91$.

(30) Guo, X.; Waser, R. Prog. Mater. Sci. 2006, 51 (2), 151. doi: 10.1016/j.pmatsci.2005.07.001

(31) Wang, X. D.; Mab, Y.; Raza, R.; Muhammed, M.; Zhu, B. Electrochem. Commun. 2008, 10, 1617. doi: 10.1016/j. elecom.2008.08.023
(32) Zhang, Z.; Zhang, H.; Liu, C. F.; Liu, Y. L.; Li, Z. C. Materials Science and Engineering of Powder Metalluray 2011, 16 (5), 682. [张 哲, 张 鸿, 刘超峰, 刘玉龙, 李志成. 粉末冶金材 料科学与工程, 2011, $16(5), 682$.]

(33) Guo, X.; Sigle, W.; Maier, J. J. Am. Ceram. Soc. 2003, 86, 77. doi: 10.1111/jace.2003.86.issue-1

(34) Zhang, T. S.; Ma, J.; Chan, S. H.; Kilner, J. A. Solid State Ionics 2005, 176 (3-4), 377. doi: 10.1016/j.ssi.2004.07.022

(35) Schouler, E.; Giroud, G.; Kleitz, M. J. Chem. Phys. 1973, 70, 1309.

(36) Rahmawati, F.; Prijamboedi, B.; Soepriyanto, S.; Ismunandar. Int. J. Min. Met. Mater. 2012, 19 (9), 863. doi: 10.1007/s12613012-0640-0

(37) Cho, Y. H.; Cho, P. S.; Auchterlonie, G.; Kim, D. K.; Lee, J. H.; Kim, D. Y.; Park, H. M.; Drennan, J. Acta Materialia 2007, 55 (14), 4807. doi: 10.1016/j.actamat.2007.05.001

(38) Kim, D. K.; Cho, P. S.; Lee, J. H.; Kim, D. Y.; Park, H. M.; Auchterlonie, G.; Drennan, J. Electrochem. Solid State Lett. 2007, 10 (5), 91. doi: 10.1149/1.2710959 